\title{
Puissance syntaxique et subtilité vocale, Claude Simon traduit en anglais
}

\section{Peter France}

\section{(2) OpenEdition \\ 12 Journals}

Édition électronique

URL : http://journals.openedition.org/ccs/943

DOI : $10.4000 /$ ccs. 943

ISSN : 2558-782X

Éditeur :

Presses universitaires de Rennes, Association des lecteurs de Claude Simon

\section{Édition imprimée}

Date de publication : 30 avril 2015

Pagination : 47-59

ISBN : 9782753539990

ISSN : $1774-9425$

\section{Référence électronique}

Peter France, «Puissance syntaxique et subtilité vocale, Claude Simon traduit en anglais », Cahiers Claude Simon [En ligne], 10 | 2015, mis en ligne le 22 septembre 2017, consulté le 07 mai 2019. URL http://journals.openedition.org/ccs/943; DOI : 10.4000/ccs.943 


\title{
PUISSANCE SYNTAXIQUE ET SUBTILITÉ VOCALE, CLAUDE SIMON TRADUIT EN ANGLAIS
}

\author{
Peter FRANCE \\ University of Edinburgh
}

\section{CLAUDE SIMON EN ANGLAIS: UNE VUE D'ENSEMBLE}

En dépit du prestige associé au Prix Nobel, les romans de Claude Simon sont restés assez peu connus dans les pays anglophones. Simon a partagé à cet égard le sort de la plupart des romanciers français du dernier demi-siècle. Dans l'immédiat après-guerre, le roman français gardait quelque chose de son ancien prestige, et les lecteurs et traducteurs américains et britanniques se précipitaient sur les œuvres de Camus, Sartre, Malraux, Beauvoir, Saint-Exupéry ou Simenon. On ne saurait en dire autant de leurs successeurs ${ }^{1}$. Ceux qu'on peut appeler les Nouveaux Romanciers (Robbe-Grillet, Butor, Sarraute et surtout Duras) étaient certes connus - et souvent admirés - par le public cultivé et les spécialistes, qui en général pouvaient les lire en français; on sait que quelques-unes des meilleures monographies sur Simon sont dues aux simoniens britanniques de la première heure ${ }^{2}$. Mais la plupart des anglophones ont du mal à lire un roman français dans le texte et à quelques exceptions près, les

1. Voir Marilyn Gaddes Rose, "Twentieth-Century French Fiction ", dans The Oxford Guide to Literature in English Translation (dir. P. France), Oxford, Oxford University Press, 2000, p. 287-292). Dans cette perspective, la critique affirme: "there have been many other French authors, much read in French and translated into English rather promptly and usually well, but little noticed in English. Once published, these books are quickly remaindered."

2. Jusqu'en 1990, on peut citer parmi d'autres Stuart Sykes, Les Romans de Claude Simon (Paris, Minuit, 1979); A. C. Pugh, Simon: Histoire (London, Grant and Cutler, 1982); Celia Britton, Claude Simon: Writing the Visible (Cambridge, Cambridge University Press, 1987). 
traductions des romanciers contemporains n'atteignaient guère le grand public, ce qui reste le cas jusqu'à nos jours.

Considérons brièvement l'histoire des romans de Simon en anglais. Cela commence en 1959, avec la publication à New York de The Wind, excellente traduction du Vent par Richard Howard, qui par la suite traduira avec brio tous les grands romans des années suivantes: The Grass (1960), The Flanders Road (1961), The Palace (1963), Histoire (1967), The Battle of Pharsalus (1971). Comme on le voit, la publication des traductions suit d'assez près la parution des textes français - Simon est porté par l'actualité littéraire du Nouveau Roman. Par la suite, il y aura quelquefois des intervalles plus longs - The Georgics, traduction de Beryl et de John Fletcher, ne paraîtra qu'en 1989, huit ans après Les Géorgiques. Mais tout compte fait, la traduction de l'œuvre se poursuit de façon régulière; les traducteurs sont en général hautement qualifiés, et tous les textes importants sont concernés.

Mais que deviennent ces traductions? Après la première publication des deux côtés de l'Atlantique, elles deviennent souvent difficiles à trouver. Lorsque l'attribution du Prix Nobel à Simon est annoncée en 1985, presqu'aucun de ses romans n'est disponible en libraire, et son éditeur américain Braziller se hâte de ressortir les traductions d'Howard. Même après le Nobel, la situation ne change pas vraiment. Prenons l'exemple de L'Herbe. En préparant la présente communication, j'avais besoin de lire la traduction de Howard, publiée pour la première fois en 1960, rééditée en 1986. Habitant Edimbourg, je pouvais en principe consulter l'exemplaire acquis par la Bibliothèque Nationale d'Écosse en tant que bibliothèque de dépôt, mais malheureusement, il était indisponible. Les autres bibliothèques publiques ou universitaires écossaises ne possédant pas un seul exemplaire de cet ouvrage, il fallait la chercher dans une des grandes bibliothèques anglaises (le site COPAC en nomme sept en tout). Qui plus est, ce texte est absent des librairies, même en ligne. La seule manière d'obtenir un exemplaire aurait été de le commander à un bouquiniste aux États-Unis, en passant par le site AbeBooks.

Dans ces conditions, il va sans dire qu'aucune retraduction d'un roman de Simon n'est venue concurrencer les traductions existantes, pour la plupart de très bonne qualité d'ailleurs. Les rééditions sont rares. Tout au plus remarque-ton une nouvelle édition de Flanders Road de Richard Howard dans la collection One World Classics en 2010 - mais rien dans les grandes collections populaires telles que Penguin Classics. Il y a là de quoi décourager les nouvelles initiatives, mais on sait que l'édition britannique et américaine n'accueille pas volontiers les 
traductions, qui ne constituent qu'une proportion très réduite de la production littéraire (on cite parfois le chiffre de 3 pour cent) ${ }^{3}$.

Ceci dit, les traductions de Simon en anglais offriraient sans doute un champ d'investigation passionnant. Cependant, plutôt que m'y aventurer, j'ai pensé qu'il serait plus intéressant ici de mettre la main à la pâte, en essayant de traduire moimême quelques pages de Simon pour ensuite proposer quelques remarques sur les différentes questions soulevées par cette tentative. Il faut insister d'abord sur le fait que je ne suis aucunement un traducteur professionnel du roman français. Si j'ai publié des traductions, il s'agit plutôt de textes classiques français (Diderot, Rousseau) ou de poésies russes (dernièrement, Lermontov, Baratynski, et Mandelstam). Mais je suis un lecteur passionné de Simon depuis ma découverte de La Route des Flandres en 1960, et j'ai beaucoup réfléchi à sa vision et à sa manière de la communiquer au lecteur. Une traduction des premières pages de L'Herbe est donnée en annexe; le lecteur intéressé pourra la comparer avec la version classique de Richard Howard dans The Grass.

\section{QUESTIONS DE FIDÉLITÉ}

Pour mieux situer Claude Simon par rapport au roman de langue anglaise, John Fletcher comparait naguère "chacun de ses romans les plus importants à l'œuvre d'un confrère [par exemple, V. S. Naipaul, Angus Wilson, Alexandre Soljenitsyne] avec laquelle il possède un certain rapport thématique ${ }^{4}$ ». Il est indéniable qu'on voit chez Simon bien des éléments - thèmes, personnages, histoires, décors - qu'on trouve dans des romans de facture plus " traditionnelle ". Mais je doute fort qu'en le traduisant en anglais on soit tenté de remanier ses textes de façon à les faire entrer dans les cadres habituels du roman de langue anglaise - ordre chronologique et logique, transitions imperceptibles ou bouleversantes, phrases plus courtes, syntaxe et ponctuation classiques. Car il va de soi que l'essence de Simon réside dans sa manière d'écrire; sa vision du monde passe par une disposition inhabituelle des matériaux du récit, par une syntaxe très personnelle qui donne naissance à un rythme très particulier. Et c'est cette disposition, cette syntaxe, ce rythme que le traducteur essaiera de conserver, en résistant à la tentation de les adapter aux normes de la culture cible.

3. Voir Anthony Pym, «Late Victorian to the Present », dans Oxford Guide., p. 73-81 : «In general, then, other languages translate from English, but English receives relatively few translations from anywhere " (p. 80).

4. John Fletcher, Claude Simon and Fiction Now, Londres, Calder and Boyars, 1975, p. 14. 
La dernière phrase de notre texte nous en offre un exemple saisissant. Après l'interruption brusque du long discours de Louise, Simon évoque un chant d'oiseau qui semble la relayer, mais cette évocation est aussitôt interrompue - entre sujet et verbe - par une longue parenthèse fort complexe:

puis, aussi brusquement, le chant - une brève série de notes redoublées, comme une arabesque calligraphiée, s'enroulant très vite plusieurs fois sur elle-même dans la même boucle compliquée, puis s'échappant, s'élevant, s'étirant dans un long et péremptoire paraphe arrêté net $\operatorname{cessa}^{5} \ldots$

En lisant cette phrase, le traducteur ressent le choc provoqué par cette rupture de la syntaxe normale; s'il voulait à tout prix rendre le texte plus «lisible", il pourrait sans doute réarticuler la phrase pour éviter cette séparation si éloquente du sujet et du verbe - et il perdrait ainsi toute la force du texte. Ici, la tâche du traducteur est facile à définir.

Il n'en va pas tout à fait de même pour la répétition. Lorsqu'un auteur vise un effet particulier en répétant plusieurs fois le même mot ou la même expression, il semblerait normal que le traducteur lui emboîte le pas. Ainsi, dans les premières dix-sept lignes de notre texte le mot "rien" revient cinq fois: " elle n'a rien... elle ne t'est rien... elle ne t'est rien... Alors rien... Rien: elle ne s'est jamais mariée. " J'ai tenu à garder la même répétition : "she has nothing... she's nothing to you... she's nothing to you... Then nothing... Nothing, she never got married. » Richard Howard par contre a sans doute pensé que "she's nothing to you " ne rend pas avec assez de précision le sens du français, et a préféré sacrifier la répétition à une traduction peut-être plus exacte: "she's not related to you ".

En revanche, dans le premier paragraphe du long discours de Louise, j'ai à mon tour cédé à la tentation de simplifier un peu la syntaxe du français. Simon coupe la phrase de Louise par une longue parenthèse qui évoque les sacrifices consentis par les sœurs pour la carrière de leur frère:

...dont elles ont réussi (à force de réfléchir au meilleur moyen de porter une robe à peu près trois fois plus de temps qu'il n'en faut pour s'user jusqu’à la trame au tissu dont elle a été primitivement faite) à faire un professeur de Faculté... (p. 9).

Trop sensible à la maladresse de cette construction (maladresse de Simon? maladresse de Louise?), j'ai tenté d'alléger un peu la phrase (" by working out the best way to make a dress last about three times as long as it takes for the original fabric to become threadbare »). Aurais-je mieux fait de conserver la prolixité de l'original?

5. Claude Simon, L'Herbe, Minuit, 1958, p. 13. 


\section{COULEUR LOCALE, UNIVERS DISCURSIF}

Dans sa vision du monde, dans son choix de personnages, dans le décor de ses romans, Claude Simon me paraît un auteur très français. Ses personnages appartiennent à un "univers discursif " (universe of discourse, pour emprunter la terminologie du grand traductologue André Lefevere $)^{6}$, qui risque de dépayser tant soit peu le lecteur anglophone. Cela ne pose pas en soi de grands problèmes au traducteur, qui peut facilement évoquer ce monde légèrement exotique; on sait que le mot bread ne désigne pas tout à fait le même produit que le mot pain, mais le lecteur britannique a sans doute assez d'expérience d'une culture voisine pour mettre une baguette ou une miche à la place d'un bloomer ou d'un cob.

Il existe cependant des concepts ou des objets qu'il est difficile de faire comprendre à un public peu au fait de la vie française. Dans un texte romanesque on hésite à imposer au lecteur un appareil fastidieux de notes en bas de page; on est obligé donc de faire entrer l'explication dans le texte. Parfois, on est tenté de garder l'expression française pour souligner les différences de culture. Ainsi, Richard Howard traduit "professeur de Faculté " par "Professeur de Faculté ». Il n'est pas certain que le lecteur américain comprenne l'expression, même si aux États-Unis le personnel enseignant des universités s’appellent les faculty members. En revanche, le university professor que j'ai préféré pourrait sembler un personnage un peu trop huppé.

Si les professeurs de faculté ont à peu près les mêmes fonctions des deux côtés de la Manche, les instituteurs - si importants dans le roman de Simon - ne sont pas tout à fait nos primary school teachers; tout un mythe républicain les en sépare, mythe que les deux sœurs Marie et Eugénie incarnent à leur manière. Il serait souhaitable de pouvoir garder le mot instituteur ou institutrice (en italique) - mais on ne serait pas compris. Force est donc de se contenter du simple mot teacher, en espérant que le contexte rendra compte de la spécificité de l'école primaire républicaine et laïque.

$\mathrm{Si}$, dans le texte que j'ai traduit, il y a un fait de civilisation peu accessible aux anglophones, c'est bien "le globe, la couronne de mariée " que Louise dit avoir cherché des yeux en entrant chez sa tante. On lit sur le site web de l'Écomusée des Vieux Métiers que « la coutume de mettre couronne, bouquet et autres souvenirs de mariage sous verrine (ou globe) s'est limitée à la France et a concerné essentiel-

6. André Lefevere, Translation, Rewriting, and the Manipulation of Literary Fame, Londres et New York, Routledge, 1992, p. 87-98. "Lefevere définit le "universe of discourse" comme "objects, concepts, customs belonging to the world that was familiar to the writer of the original" ". 
lement les catholiques ". Mais heureusement ici, même si le lecteur n'a jamais vu un globe de mariée, il est relativement facile de représenter cet objet en traduisant l'expression de Simon par "the glass case with the bridal crown "; le contexte permet de comprendre la signification de cette couronne.

Il semble bizarre de transposer brutalement l'action d'un roman simonien vers un milieu trop clairement britannique ou américain, comme l'a fait Helen R. Lane dans sa traduction de Triptyque où l'affiche "GALERIES MODERNES... LE CHIC, HOMMES-FEMMES " devient: "BROADWAY DEPARTMENT STORE - THE CITY'S NEWEST... LATEST FASHIONS 7 . » Mais pour ceux ou celles qui traduisent vers l'anglais, la notion de universe of discourse soulève fatalement le problème des variétés de la langue anglaise et des différentes sociétés qu'elles reflètent - et surtout celui de l'écart entre l'anglais du Royaume-Uni et celui des États-Unis (dans les deux cas, bien entendu, il y a des variantes innombrables de la langue nationale, dont quelques-unes, l'écossais par exemple, ont acquis un certain droit de cité dans le monde littéraire). On a dit qu'il s'agit de deux cultures séparées par une langue commune, et il est vrai que pour certains realia le traducteur est obligé de choisir - s'il écrit corncela signifie le maïs pour les américains, le blé pour les britanniques, tandis que lepavement est la chaussée aux États-Unis, le trottoir en Grande-Bretagne. Dans ces conditions les éditeurs préfèrent naturellement aligner le lexique, la syntaxe et l'orthographe des traductions sur les normes de leur pays.

Dans le cas qui nous occupe, il n'y a pas de difficulté majeure. La langue de Richard Howard ne choque aucunement un lecteur britannique; dans les quatre pages en question, il n'y a que deux ou trois éléments de vocabulaire qui produisent un léger effet de dépaysement: figuring out, stock-raisers, cookie jar... Le dernier exemple est intéressant. Simon parle d'une "boite à biscuits ou à berlingots, en fer", ce que j'ai traduit par " a biscuit tin or a sweetie tin " (le fer est impliqué par le mottin); Howard garde le "biscuit tin", mais pour les berlingots, il choisit " a cookie jar made out of tin ", où le lecteur britannique sent une contradiction entre le jar (fait de verre au Royaume-Uni) et le fer-blanc. Quant aux berlingots, le véritable équivalent dans la culture anglaise serait sans doute le humbug, mais ce mot ferait surgir un monde trop spécifiquement britannique; j'ai donc préféré lesweetie tout en sachant que ce mot donne au texte un ton quelque peu enfantin (mais aussi écossais). On ne peut pas éviter le dépaysement

7. Claude Simon, Triptyque, Minuit, 1973, p. 209; Triptych, traduit par Helen R. Lane, London, John Calder, 1986 (première édition 1977), p. 159. 
- c'est une entreprise bizarre de faire revivre les personnages de Simon dans un monde qu'ils n'ont jamais connu.

\section{LES VOIX DU TEXTE}

La syntaxe spécifique de Claude Simon pourrait sembler poser des problèmes au traducteur: comment reproduire dans une autre langue ce flux de paroles construit sur des répétitions, des appositions, des accumulations, des changements abrupts de direction? En réalité, pour l'anglais au moins, la tâche n'est pas trop difficile. Il s'agit en général de suivre le mouvement de la phrase aussi fidèlement que possible - tous les éléments que je viens d'énumérer peuvent être utilisés en anglais, et produisent sensiblement le même effet qu'en français, fondé sur l'écart de ce style par rapport à la syntaxe habituelle de la narration. Même le participe présent, cheville ouvrière de la phrase simonienne, s'emploie de la même façon, comme dans l'exemple suivant, où la syntaxe française est reproduite assez exactement:

... the voice falling silent, breaking off abruptly, and Louise remaining there, panting a little, as if surprised and furious at having talked so much, still looking at the something that he could not see - that he knew he could not see and would never see, even if he turned and looked over his own shoulder to where the something seemed to be...

Ce qui m'a semblé plus délicat, c'est de faire entendre en anglais les diverses voix du texte simonien. Dans L'Herbe, on entend plusieurs voix, celle de Louise, celle de Sabine, celle du médecin et ainsi de suite... mais aussi la voix d'un narrateur dont on ne sait rien d'autre. Souvent, dans la fluidité du texte, il est difficile de saisir les changements vocaux, de savoir qui parle, qui pense, qui voit. Comment distinguer, par exemple, la voix, la pensée et les perceptions de Louise, de Sabine et du narrateur dans le passage suivant:

...et maintenant Louise pouvait entendre à travers la cloison les bribes d'un calcul compliqué, Sabine spéculant apparemment sur le probable état de décomposition avancé des plus anciens cadavres de la famille (qu'elle dénombrait), putréfaction et même, espérait-elle, pulvérisation qui permettrait sans doute - comme elle avait entendu dire que cela avait été fait une fois dans le caveau d'une famille amie et trop nombreuse - de réunir plusieurs débris dans un seul et collectif cercueil... (p. 123).

Dans les quelques pages qui nous concernent ici, on entend en principe trois voix, celles de Louise et de son interlocuteur (dont on ne sait rien pour l'instant) et celle du narrateur, voix encore discrète, mais qui s'affirme vers la fin du passage pour évoquer aussi bien les actions et les sensations de Louise que le monde qui 
l'entoure. Le problème, c'est que dans les longs discours de Louise on semble s'éloigner assez rapidement d'une parole réaliste vers des considérations plus complexes, exprimées dans un style qui appartient plutôt au narrateur qu'au personnage (voir surtout les paroles sur le "temps accumulé »). Dans le paragraphe qui commence: "Alors rien " ("Then nothing "), par exemple, la déclaration initiale: "elle ne s'est jamais mariée " est aussitôt suivie par des réflexions sur les rapports des deux sœurs et de leur jeune frère qui semblent représenter la pensée (de Louise, ou du narrateur) plutôt que la parole directe. La voix qui raconte réapparaît avec des expressions comme " et quand nous nous sommes mariés ", " elle m’a fait venir dans ma chambre ", ou " et alors elle a fouillé ", mais cette narration de base est noyée dans des digressions et des parenthèses. Vers la fin du paragraphe, on arrive à une description minutieuse de la boîte à berlingots d'un type très familier au lecteur de Simon, surtout lorsque l'image de la jeune femme est répercutée dans une suite d'images identiques, "comme dans ces jeux de miroirs sans fin". Ici et ailleurs, le passage au présent creuse un fossé entre la description et le récit fait par Louise à son ami, soulignant ainsi l'écart entre personnage et narrateur :

...une boite à biscuits ou à berlingots, en fer, toute piquetée de rouille avec, dessus, une jeune femme vêtue d'une longue robe blanche, à demi allongée sur l'herbe dans une pose à la fois langoureuse et raide, avec juste la pointe des pieds, ou plutôt des souliers, dépassant sous le dernier volant, pudiques et ridicules, et, couché près d'elle (qui dans sa main tient une même boîte sur le couvercle de laquelle sa même image se répète, comme dans ces jeux de miroirs sans fin) un de ces petits chiens blancs et frisés... (p. 10).

Le traducteur s'efforcera de suivre les méandres du texte, mais peut-il faire ressortir en anglais la distinction entre la voix de Louise et celle du narrateur? Dans le texte français, il y a certains marqueurs du français parlé, des expressions un peu familières comme "ça ne va pas ensemble ", et surtout l'emploi du passé composé ("Elle ne m’a rien demandé et elle m’a donné cette bague, elle m’a aimée »), que l'on fera entrer en contraste avec le passé simple du narrateur, en particulier le mot « cessa " qui renvoie au chant de l'oiseau à la fin de cet extrait. En anglais, les temps du verbe ne marquent pas ainsi des registres distincts de langue, mais on peut obtenir un effet comparable en utilisant des contractions du type he's (pour he is) ou couldn't (pour could not), qui appartiennent plutôt au registre parlé qu'au récit littéraire. C'est pourquoi j'ai tenu à éviter les contractions dans le récit du narrateur, tout en les employant librement pour les paroles qui sont censées être prononcées par les personnages. Ainsi, au début de mon texte anglais, Louise se sert des expressions "there'll be no-one ", "what's death ", " he's old " et " he won't weep ", tandis que le narrateur dit (ou écrit): "she kept looking straight 
ahead at something he could not see ". Ce could not, d'un effet un peu lourd sans doute, devrait en principe attirer l'attention du lecteur sur le changement de voix. Mais cela risque de passer inaperçu, le contraste de tons n'étant pas assez fort.

Que dire en guise de conclusion? Tout en regrettant l'absence de visibilité de Simon dans les pays de langue anglaise, il faut souligner que la qualité des traductions n'est pas en cause. Richard Howard en particulier a fait un travail remarquable, ses traductions sont à la fois fidèles et entraînantes, et pour l'instant il n'y aurait aucune raison d'essayer de remplacer ses textes sur le marché littéraire en Grande-Bretagne ou aux États-Unis. En traduisant pour le simple plaisir, cependant, comme je l'ai fait, on apprend à mieux lire et à mieux apprécier le travail de ce maître qu'est Claude Simon. 


\section{ANNEXE}

\section{GRASS - CLAUDE SIMON}

\section{traduit en anglais par Peter France}

"But she has nothing, no-one, and there'll be no-one to weep for her (and what's death without the weeping?), no-one but her brother perhaps and he's old too and probably he won't weep for her any more than she would weep for herself, I mean would allow herself to weep, would think it was decent, proper to..."

"But she's nothing to you."

"No."

"She's nothing to you."

"No", she repeated meekly. But she kept looking straight ahead at something he could not see.

"Well then."

"Then nothing", she said (still looking beyond the trees, the meadows, the peaceful September countryside at the something he could not see). "Nothing; she never got married. Maybe it never occurred to her that she could, that she had the right - her with a brother fifteen years younger than her and brought up by the two of them (her and the one who's dead), whom they managed (by working out the best way to make a dress last about three times as long as it takes for the original fabric to become threadbare) to turn into a university professor, which for two primary school teachers whose father and mother could barely read or maybe not even, must have seemed worth the sacrifice of everything a woman can normally expect, and when we got married, Georges and I, she gave me this ring, she called me into her room (and that was the first time I noticed that smell, just like a dried rose or rather - since a dried rose has no smell - the smell that you imagine it might have, in other words something both dusty and fresh at the same time, and I looked at her table, her dressing table, but there was nothing on it but these four pins and this bottle of cheap eau de cologne, and yet it smelt like a flower, like a young girl, or how the room, or rather the tomb, the sarcophagus of a very young girl might smell if she had been preserved there, intact, but ready to crumble to dust at the least breath of air), and then she rummaged in a drawer and pulled out not a jewel box or even one of those steel boxes that ironmongers sell to peasants and cattle dealers who don't want to put their money 
in the bank, but a biscuit tin or a sweetie tin, all spotted with rust, and on its lid a young woman wearing a long white dress, half stretched out on the grass in a pose that is both languid and stiff, with just the tips of her toes, or rather her shoes, sticking out, modest and ridiculous, from under the bottom petticoat, and lying beside the woman (who is holding in her hand another tin with a lid showing the same picture, like endlessly repeating mirrors) one of those little white curly dogs, the whole thing (lady, poodle, meadows) in a frame of flowers and ribbons with periwinkle blue bows, and...

"But...

"No, listen: there was no key of course and the box was only fastened by a long string wound round it twenty times or more, so she took some time to unwind it, then clutched the box to her body while her stiff clumsy fingers struggled to open it - and all the time I was sensing that smell of a young girl, a flower, searching for the glass case with the bridal crown, searching, but there was nothing. Nothing but that intoxicating and no doubt imaginary perfume of freshness, virginity and accumulated time. Not wasted: vanquished, or rather overcome, tamed: no longer the hereditary enemy, omnipresent and omnipotent, whom you watch in terror as it advances and flows past slowly and relentlessly, but an old fellow-traveller, familiar, once feared and hated perhaps, but so long ago that the memory of fears and terrors is like that of our childhood panics which now merely raise a smile...

I know, they don't go together: a young girl, the scent of jasmine, and that body on the point of crumbling into dust, so familiar with time that it seems to be time itself, and those skinny yellow hands - polished as ivory in places - struggling with their own clumsiness and the rust on the box (as if the rust and the clumsiness were one and the same thing, always the same: the years, time) until she finally managed to open it, and then rummaging around in its contents, not sweets but odd buttons, gold (or gold-plated) chains and old brass shoe buckles, and finally handing it to me: not in a case, a casket with the name of a fashionable jeweller like the one that Georges (or rather his mother) had bestowed on me (not really me, though, but themselves, giving themselves a ceremonial gift, because he - and she - would have regarded it as a mark of dishonour for me to wear anything worth less than five hundred thousand francs on my finger... And so, even if she didn't approve of him marrying me - not that she said as much or even let it slip out - giving him the necessary five hundred thousand francs, or perhaps, to be on the safe side, going to choose it herself and only handing it to 
him at the very last moment, just before he slipped it on to my finger). No casket then, just a scrap of cotton wool.

Yes, that's the one. And I probably wouldn't even get ten thousand francs for it from a jeweller, but I wouldn't sell it for two or three times as much, or for any money. When I leave, I'll give him back (give them back - him and his mother) all their jewels; I'll pile them up on my dressing table as I go, or maybe I'll throw them in his face, not because he deserves to have them thrown in his face, but because that's the kind of gesture that helps at moments like that, but this one, I'll say: 'I'm keeping it'. Because she was the one who gave it to me. You understand? She asked nothing of me and she gave me this ring, she loved me, simply because I was Georges's wife, I could have been a tart, a duchess, a thief, she'd have loved me just the same, and without asking for anything in return. Because she never asked other people for anything, not even to love her or to allow her to love them, any more than she allowed herself to tell them so or to show it in any other way than the only way she could imagine, by giving them all she could afford, or even what she couldn't afford, finding a way of affording what she couldn't afford. That's why I stayed on and didn't leave sooner. I would have left Georges long ago, even before I met you, if it hadn't been for that. I don't even say if it hadn't been for 'her', I say: if it hadn't been for 'that'. And now she's going to die, and then there will be nothing" (the voice falling silent, breaking off abruptly, and Louise remaining there, panting a little, as if surprised and furious at having talked so much, still looking at the something that he could not see - that he knew he could not see and would never see, even if he turned and looked over his own shoulder to where the something seemed to be, and a moment later a bird started singing close by and then, just as suddenly, the song - a short sequence of double notes, like a calligrapher's scroll-work, twisting rapidly back on itself several times in succession, repeating the same complicated loop, then breaking free, rising and stretching itself in a long decisive flourish that is cut short - stopped, and again one could hear the distant and discordant racket of sparrows gathering for the night in a clump of bamboos). 


\section{ILLUSTRATION}

Une page manuscrite de la traduction des Géorgiques par John Fletcher (C. Simon, Euvres II, Bibliothèque de La Pléiade, p. 913-914). "Literary Translation Archive, University of East Anglia"

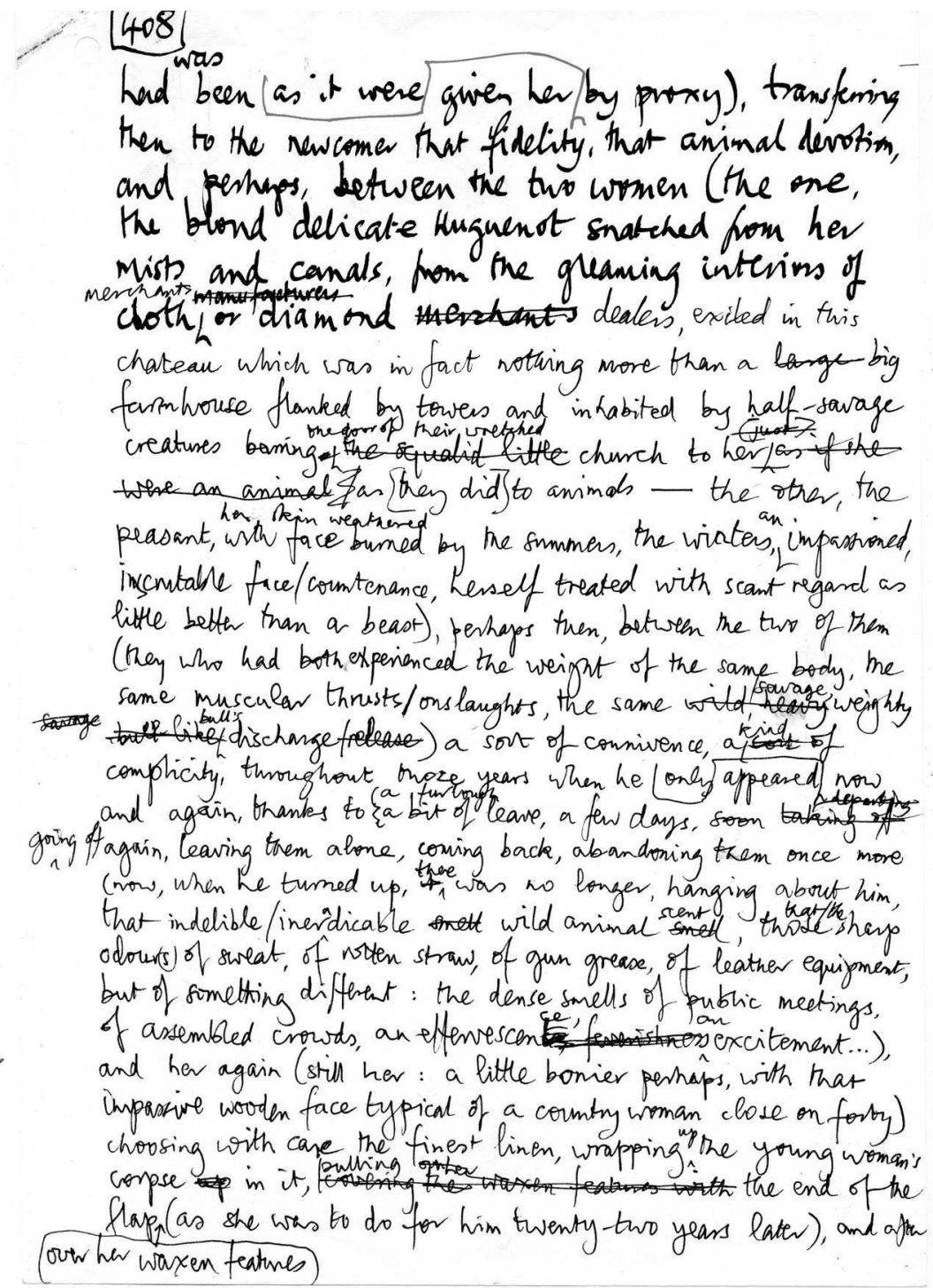

